\title{
A Survey on Gain-Scheduled Control and Filtering for Parameter-Varying Systems
}

\author{
Guoliang Wei, ${ }^{1}$ Zidong Wang, ${ }^{2,3}$ Wangyan $\mathrm{Li}^{4}$, and Lifeng $\mathrm{Ma}^{5}$ \\ ${ }^{1}$ Department of Control Science and Engineering, University of Shanghai for Science and Technology, Shanghai 200093, China \\ ${ }^{2}$ Department of Computer Science, Brunel University, Uxbridge, Middlesex, UB8 3PH, UK \\ ${ }^{3}$ NAAM Research Group, Department of Mathematics, King Abdulaziz University, Jeddah 21589, Saudi Arabia \\ ${ }^{4}$ Business School, University of Shanghai for Science and Technology, Shanghai 200093, China \\ ${ }^{5}$ School of Automation, Nanjing University of Science and Technology, Nanjing 210094, China
}

Correspondence should be addressed to Guoliang Wei; guoliang.wei1973@gmail.com

Received 4 December 2013; Accepted 28 March 2014; Published 29 April 2014

Academic Editor: Jinde Cao

Copyright (c) 2014 Guoliang Wei et al. This is an open access article distributed under the Creative Commons Attribution License, which permits unrestricted use, distribution, and reproduction in any medium, provided the original work is properly cited.

This paper presents an overview of the recent developments in the gain-scheduled control and filtering problems for the parametervarying systems. First of all, we recall several important algorithms suitable for gain-scheduling method including gain-scheduled proportional-integral derivative (PID) control, $\mathrm{H}_{2}, \mathrm{H}_{\infty}$ and mixed $\mathrm{H}_{2} / \mathrm{H}_{\infty}$ gain-scheduling methods as well as fuzzy gainscheduling techniques. Secondly, various important parameter-varying system models are reviewed, for which gain-scheduled control and filtering issues are usually dealt with. In particular, in view of the randomly occurring phenomena with timevarying probability distributions, some results of our recent work based on the probability-dependent gain-scheduling methods are reviewed. Furthermore, some latest progress in this area is discussed. Finally, conclusions are drawn and several potential future research directions are outlined.

\section{Introduction}

During the past decades, the gain-scheduling control and filtering approach has received a lot of attention from the control community. This stems from the fact that the gainscheduling method is more effective than the traditional ones to cope with the unavoidable nonlinearities and time-varying dynamics of the practical plant. Control and filtering problems are two fundamental issues in control theory and have been intensively discussed by a great number of researchers. The control problems can be classified into two types: state feedback control and output feedback control according to the controller structure. The state feedback controller is based on the state information of the systems, while the purpose of output feedback is to design a controller in terms of measurement output rather than the state of a given system. On the other hand, the general idea of filtering problems is to form a kind of "best estimate" for the true state of some certain system by some potentially noisy observations.

In fact, gain-scheduling is a broad notion that gives rise to many different design ideas, for example, precompensating a nonlinear gain with the inverse gain function, switching gain values according to operating conditions or even according to preset times, controller switching and controller blending, and so on. Therefore, the gain-scheduling idea has been extensively applied to design controllers and filters for many kinds of systems, such as nonlinear stochastic systems with time-varying parameters, T-S fuzzy systems, linear parameter-varying systems. and Markov jumping systems. For these kinds of systems, the algorithms and performance indices have benefited from the gain-scheduling ideas, such as the gain-scheduling proportional-integral-derivative (PID) method, the fuzzy gain-scheduling approach. and the $H_{\infty}$ gain-scheduling control. 
The randomly occurring incomplete information in system models has been extensively studied by a lot of researchers and many important results have been published; see for example, [1-3] and, particularly, the survey papers $[4,5]$. A flexible and effective model, Bernoulli distribution model, has been firstly introduced and employed to cope with the randomly occurring incomplete information. However, such a random occurrence model satisfying a timeinvariant Bernoulli distribution cannot accurately describe the time-varying occurring nature in spite of its effectiveness and flexibility. Consequently, a new model with timevarying probability has later been exploited to describe the randomly occurring incomplete information. Furthermore, recently, a probability-dependent gain-scheduling approach has been proposed to deal with the control and filter synthesis problems for systems with randomly occurring incomplete information.

In this paper, we mainly focus on the gain-scheduled control and filtering problems for parameter-varying systems and aim to give a survey on some recent advances in this area. We introduce some important gain-scheduling algorithms, such as gain-scheduling PID control algorithms, fuzzy gainscheduling methods, and $H_{\infty}$ gain-scheduling techniques. The parameter-varying systems to be discussed mainly include stochastic nonlinear systems, linear parametervarying systems, networked control systems, and Markov jumping systems, where the gain-scheduling approach has been proven to be effective when it comes to such systems. It should be pointed out that, due to the pervasive existence of randomly occurring phenomena, a probability-dependent gain-scheduling approach has recently been developed to deal with such problems, and the corresponding results will be reviewed in this paper. Furthermore, a series of newly published results on the gain-scheduling control and filtering problems for parameter-varying systems will be surveyed. Finally, some conclusion remarks are drawn and several related future research directions are pointed out.

The remainder of this paper is outlined as follows. In Section 2, some existing gain-scheduling approaches are reviewed. Section 3 covers the recent developments of several important system models with time-varying parameters and Section 4 specifically addresses the probability-dependent gain-scheduled control and filtering problems for systems with randomly occurring incomplete information. Several latest results on gain-scheduled control and filtering problems can be found in Section 5. In Section 6, both conclusions and future research topics are given.

\section{Gain-Scheduled Control and Filtering}

2.1. Gain-Scheduled PID Control. In control area, PID control strategy offers a simple yet efficient solution to many realworld control problems and has become the most widely used control method [6]. Therefore, in practical systems, PID control algorithm has been employed in more than $90 \%$ of control loops. During the past decades, much attention has been paid to improve PID control performance from tuning rules, identification schemes, adaptation techniques, and so forth. At the same time, with some advanced ideas having been put forward in science fields, such as optimization algorithm [7-9], the concept of cloud computation [10-12], integration and modularity [10,13], PID technology appears to be focused on the integration of available methods in the form of software so as to get the best performance of PID control [6]. The PID controllers in the literature can roughly be divided into two main categories according to their structures. The first one is with fixed controller parameters during the control process after they have been tuned. For this category, the controllers are simple but cannot always effectively control the systems with time-varying parameters. The other one has controller gains that can be scheduled on-line based on the time-varying parameters in the plant model, which is called the gain-scheduled PID control [6]. Gain-scheduling technique is an important improvement way for PID controller structure in engineering to deal with some complex characteristics, such as nonlinear process and time-varying features. During the past decades, the gainscheduled PID control problems have obtained considerable attention and a variety of results have been reported; see for example, [14-17]. In [14], a systematic approach has been proposed to design the gain-scheduled PI controllers for nonlinear processes, which are based on the empirical stateaffine models of the process that can be directly identified from the process data. In [15], a PI-type compensator has been designed by the gain-scheduling technique, and an algorithm has been firstly presented by which the resulting compensators at fixed values of the parameter are optimally tuned. [16] has addressed the problem of the gain-scheduled PID controller design which can ensure the stability for the closed-loop systems. In the gain-scheduled controller design procedure, the maximal rate value of the gain-scheduled parameter changes has been considered, which allows us to decrease the conservativeness and obtain the controller with a given performance.

2.2. $H_{2}, H_{\infty}$, and Mixed $H_{2} / H_{\infty}$ Gain-Scheduled Approach. Control performance criteria are key elements in control theory. The most fundamental control objectives are quite naturally and effectively expressed as the norm of certain signals in the control loops. The $\mathrm{H}_{2}$ performance criterion can generally be expressed as minimizing the $\mathrm{H}_{2}$ norm of the closed-loop transfer function and internally stabilizing the system, while the objective of $H_{\infty}$ performance criterion is to obtain a controller/filter that minimizes the maximum norm (i.e., the $H_{\infty}$ norm) of an input-output operator [18]. As is well known, the $H_{\infty}$ performance is an important index for evaluating the disturbance rejection attenuation property. Since Zames's original work [19] has been published in 1981, the past two decades have witnessed a tremendous advance that has happened in the $H_{\infty}$ control theory. The standard $H_{\infty}$ control problem has been completely solved by Doyle et al. for the linear systems by deriving simple state-space formulas for all controllers [20].

Mixed $\mathrm{H}_{2} / \mathrm{H}_{\infty}$ performance can enjoy the advantages of both $H_{2}$ and $H_{\infty}$ performance. In [21], the mixed $H_{2} / H_{\infty}$ 
approach has been firstly proposed as a way to formulate an optimization problem to the standard $H_{\infty}$ control problem by using the Lagrange multiplier method. The mixed $H_{2} / H_{\infty}$ control problems have been addressed in [22], and in [23], both the state feedback and output feedback problems have been converted into convex optimization problems in the content of mixed $\mathrm{H}_{2} / \mathrm{H}_{\infty}$ control.

Under guaranteed $\mathrm{H}_{2}, \mathrm{H}_{\infty}$ or mixed $\mathrm{H}_{2} / \mathrm{H}_{\infty}$ performance, many gain-scheduled control and filtering problems are considered. In [24], the $\mathrm{H}_{2}$ gain-scheduled state feedback control problem has been considered by using the parameterdependent Lyapunov function for linear parameter-varying system. In [25], the $H_{\infty}$ gain-scheduled controllers for timevarying systems have been designed. Both generalized $\mathrm{H}_{2}$ and $H_{\infty}$ discrete-time gain-scheduled filter design problems have been investigated in [26] with the results extended to mixed $H_{2} / H_{\infty}$ designs. In [27], it has been shown that an $H_{\infty}$ gain-scheduled controller can be obtained for a finite set of disturbance frequencies by convex optimization method. [28] has considered the $H_{2}$ and $H_{\infty}$ performance analysis and synthesis procedures for the design of both gain-scheduled and robust static output feedback controllers as well as the mixed $\mathrm{H}_{2} / \mathrm{H}_{\infty}$ guaranteed cost control problem.

2.3. Fuzzy Gain-Scheduling Method. Many mathematical models for real-world phenomena are inherently nonlinear, and the stability analysis and synthesis problems for nonlinear systems are generally difficult. To facilitate the mathematical analysis, in the literature, some stringent assumptions have been imposed on the nonlinearities, such as smoothness and Lipschitz continuity, which have inevitably led to considerable conservatism. As an alternative approach, in the past few decades, the fuzzy logic theory has been demonstrated to be effective in dealing with a variety of complex nonlinear systems, which has therefore received a great deal of attention in the literature; see for example, [29-32]. The fuzzy controller consists of a set of fuzzy control rules, each of which is derived from the corresponding rule of the process model. Among various fuzzy systems, one of the most popular models is the Takagi-Sugeno (T-S) model; see [29, 33-35] for some recent publications. In this type of fuzzy model, a nonlinear system is represented by a set of local linear models smoothly connected by nonlinear membership functions, which have a convenient and simple dynamic structure and hence the existing results for linear systems can be readily extended to this class of nonlinear systems.

The fuzzy controller can be described as

$$
u_{k}=\sum_{i=1}^{r} h_{i} K_{i} x_{k},
$$

where $x_{k}$ is the state variable of the fuzzy system, $r$ is the number of fuzzy rules, $K_{i}$ is the controller gain for the rule $i, u_{k}$ is the control input, and $h_{i}$ is the fuzzy weight obtained in the fuzzy model of the controlled system. Note that different control rules are with an identical structure but different gains. Therefore, such a fuzzy controller is referred to as a fuzzy gain-scheduled controller, where gain- scheduling approach is performed by the fuzzy weights $h_{i}(i=1,2, \ldots, r)$. Up to now, many results on the fuzzy gainscheduled control problem have been published; see [36-38].

In [36], the development of a fuzzy gain-scheduling scheme of PID controllers is described for the process control. Fuzzy rules are utilized on-line to determine the controller parameters based on the error signal and its first difference. Simulation results demonstrate that better control performance can be achieved in comparison with the Ziegler-Nichols controllers and the Kitamoris PID controllers. In [37], a model-based fuzzy control problem has been addressed, and a constructive and automated method for the design of a gain-scheduled controller is presented. Based on a given T-S fuzzy model of the plant, the controller is designed such that stability and prescribed performance of the closed loop are guaranteed. In [38], the robust $H_{\infty}$ control problem has been investigated for a class of uncertain time-delay T-S fuzzy systems, where the delays are assumed as infinite-distributed delays and the uncertain parameters are norm-bounded. By using the Lyapunov stability theory and the linear matrix inequality (LMI) technique, sufficient conditions have been developed so that the closed-loop fuzzy control system is guaranteed to be exponentially stable with $H_{\infty}$ performance.

\section{Systems Models}

This section gives a systematic overview of recent advances on several common systems, for which the gain-scheduling technique is suitable to design controllers and filters. In general, these systems can be categorized as linear parameter-varying systems, stochastic nonlinear systems, networked control systems, and Markovian jump systems. In the following, we will take a deep investigation of these systems one by one in order to inspire more research interest.

3.1. Linear Parameter-Varying Systems. Linear parametervarying (LPV) systems are a very special type of systems, whose state-space system matrices are functions dependent on unknown but measurable time-varying parameters, and the measurements of these parameters provide real-time information according to the variations of the plant's characteristics. In the past few years, the research on LPV systems has become a promising work from both theoretical and engineering viewpoints. For example, in [39], the sensorfault-tolerant control problem for LPV systems has been considered, while the analysis and synthesis issues have been conducted in [40] for LPV systems with parameter-varying time delays. Moreover, the designs of robust controller/filter for LPV systems have also acquired a great deal of attention, and some representative results have appeared in [41-45].

It should be noticed that, in order to design an appropriate controller/filter for the LPV systems, the gain-scheduling approach has been proven to be an effective one in this process. The idea of gain-scheduling approach is to design 
controller/filter gains as functions of the scheduling parameters, which are supposed to be available in real time and can be utilized to adjust the controller/filter with hope to get the best performance of the system. Therefore, gain-scheduled control and filtering problems for LPV systems have stirred a great deal of interest in these years; see, for example, [4648]. Survey [49] has shown in great detail about how gainscheduling approach has worked in LPV systems and several successful applications have been proposed.

On the other hand, for the purpose of designing a controller/filter with less conservatism for the LPV systems, it is natural to construct novel Lyapunov functions with scheduling parameters, which are usually called parameterdependent Lyapunov functions. Very recently, the parameterdependent Lyapunov function approach has been applied in the gain-scheduled control/filtering problems so as to achieve better control/filtering performance requirements and some results have been reported in the literature $[46,48,50,51]$.

3.2. Stochastic Nonlinear Systems. Owing to pervasive existence of stochastic perturbations in reality, stochastic models have been successfully utilized to describe many practical systems such as mechanical systems, economic systems, and biological systems. Over the past few decades, the study of stabilization, control, and filtering problems for stochastic systems has been paid much attention by many researchers and a large number of results have been obtained in the literature; see, for example, [52-56].

Besides, since nonlinearity is inevitable in most realworld systems, it is not surprising that analysis and synthesis of stochastic nonlinear systems have attracted increasing research attention, and some latest results have been published; see, for example, [57-63]. Among them, the nonlinearity has often been assumed to be sector-nonlinearity (also called sector-like nonlinearity), which is known to be quite general that includes the widely used Lipschitz condition as a special case. Moreover, the stochastic nonlinearity described by statistical means has drawn particular research focus since it covers several well-studied nonlinearities in stochastic systems; see, for example, $[55,64,65]$.

Recently, the gain-scheduled control and filtering problems for stochastic nonlinear systems have attracted increasing attention from a variety of engineering areas. For instance, in [66], the gain-scheduled PI tracking control problem for stochastic nonlinear systems subject to partially known jump probabilities has been studied, the gain-scheduled robust fault detection on a class of time-delay stochastic nonlinear systems has been considered in [67], and the gainscheduled $H_{\infty}$ controllers as well as gain-scheduled worstcase controllers have been introduced in $[68,69]$, separately. In addition, for stochastic nonlinear systems with randomly occurring incomplete information, the interested reader may consult [70-74] for some recent results.

3.3. Networked Control Systems. In recent years, with the various applications of networks in the complex dynamical processes such as advanced aircraft, spacecraft, and automotive and manufacturing processes, the networked control systems
(NCSs) have attracted much attention owing to low cost, high reliability, reduced weight and power requirements, simple installation and maintenance, and decreasing the hard wiring and implementation difficulties. NCSs are typically made up of sensors, actuators, and controllers, which communicate with a shared network. Review papers about NCSs can be found in [75-78].

Network-induced delay and packet dropout are key features of NCSs. Because of the devices connected to the shared medium, the transmission capacity of the communication network is usually limited, which in turn affects the number of bits or packets per second transported via the network. Consequently, the networked-induced delays and packet losses have become unavoidable and constitute the main causes for degrading the achievable performance of the networked systems. Therefore, in the past decade, the filtering and control problems for NCSs with communication delays and/or missing measurements have been extensively considered by many researchers; see, for example, [79-84].

As an important method, gain-scheduling can also be applied in the NCSs. In [85], the modeling and dynamic gain-scheduling design mechanism of the NCSs with packet losses have been investigated. Different from the existing static state feedback control results, [86] has constructed a gain-scheduling-based state feedback integral controller, where an integral action was introduced to address the nonzero disturbance issue, while [87] has synthesized a gainscheduled output feedback control for LPV systems with jumping parameters. Moreover, motivated by the packetbased control approach, [88] has proposed an offline modelpredictive control scheme based on the gain-scheduling method. This scheme is capable of actively compensating for the communication constraints, which is an impossible task for conventional control approaches, and at the same time it dramatically reduces the communication and computational costs compared with the packet-based control approaches.

3.4. Markovian Jump Systems. Markovian jump systems are the hybrid systems with two components in the state [89]. The first one refers to the mode which is described by a continuous-time finite-state Markovian process, and the second one refers to the state which is represented by a system of differential equations.

The jump systems have the advantage of modeling the dynamic systems subject to abrupt variation in their structures caused by component failures or repairs, sudden environmental disturbance, changing subsystem interconnections, or operating in different points of a nonlinear plant, which often take place in a lot of dynamics systems [8991]. Recently, the problems of stability and stabilization for uncertain Markovian jump systems with time delays have been extensively investigated; see [92-95] and references therein. On the other hand, filtering and control for Markovian jump systems with or without nonlinear disturbances have also drawn some research attention; see [96-98] for some related results. In addition, some papers have assumed partly unknown transition probabilities; see $[99,100]$. 
Gain-scheduling method can also be applied to analyze the Markovian jump systems. In [101], based on the stochastic Lyapunov-Krasovskii functional approach, a new globally exponentially stochastically stabilizable criterion has been derived for the jumping system by means of linear matrix inequalities. In [66], continuous gain-scheduling approach has been employed to design continuous nonlinear PI tracking controllers on the entire nonlinear jumping system.

\section{Probability-Dependent Gain-Scheduled Control and Filtering for Systems with Randomly Occurring Incomplete Information}

The randomly occurring incomplete information has recently raised a great deal of interest within the control community; it refers to these phenomena appearing in a random way based on a certain kind of probabilistic law which mainly caused by some environment reasons, such as random failures and repairs of the components, and intermittently switching in the interconnections of subsystems. These randomly occurring incomplete information phenomena under consideration mainly include missing measurements [102-104], randomly occurring delays [61], randomly occurring sensor saturations $[60,105]$, randomly occurring nonlinearities [51, $74]$, and randomly occurring actuator faults [106, 107]. For more details about these randomly occurring incomplete information phenomena, we refer readers to survey paper $[4,5]$. It is worth noting that, these randomly occurring incomplete information phenomena may potentially cause a lot of undesired problems that would deteriorate the system performance; hence, there is a great need to develop new strategy to deal with this problem effectively.

As we all know, the Bernoulli distribution model is perhaps the most effective one to be utilized in different systems (e.g., time-delay systems $[61,104,108]$, nonlinear systems [74, 109], and Markovian jump systems [110]) to reflect randomly occurring incomplete information. Recently, some efforts have been turned to describe the randomly occurring incomplete information with a more general model; for example, in $[55,111]$, a general model of multiple missing measurements has been investigated by using a diagonal matrix to describe the different missing probabilities for individual sensors. However, it should be mentioned that in most of the existing literature, the randomly occurring incomplete information has been assumed to satisfy a timeinvariant Bernoulli distribution or other distributions. Such an assumption, unfortunately, limits the application scope since randomly occurring incomplete information usually appears with time-varying probabilities due to the fact that the environment often changes from moment to moment in real systems. Therefore, it is much more meaningful to investigate the randomly occurring incomplete information with time-varying probabilities.

As we have introduced before, the gain-scheduling approach is one of the most popular ways to design a controller or filter, whose gains can be updated by a set of tuning parameters in order to optimize the closed-loop system's performance in time. On the other hand, the randomly occurring incomplete information often occurs with timevarying probabilities, which also can be considered as a tuning parameter for the controller or filter. Under such considerations, a novel gain-scheduling approach, namely, probability-dependent gains-scheduling approach, has been proposed to deal with systems with randomly occurring incomplete information.

It is noteworthy that by utilizing the probabilitydependent gains-scheduling approach, the designed gainscheduled controller/filter has not only the constant part but also the time-varying part which can be scheduled online according to the corresponding time-varying probability parameters; therefore, it will naturally lead to less conservatism than the conventional ones with fixed gains only. Associated with that, the probability-dependent Lyapunov functional has also been constructed in a sense that it can reduce the potential conservatism. With the development of the related research in the past several years, the probabilitydependent gains-scheduled controller/filter has turned out to be a very useful tool to cope with system with randomly occurring incomplete information.

Since firstly introduced in [70], the probabilitydependent gains-scheduling approach has recently received more and more attention, and a series of results have been reported; see, for example, [70, 71, 73, 74]. In [74], a probability-dependent gain-scheduled state feedback control problem has been addressed for a class of discretetime stochastic delayed systems with randomly occurring nonlinearities. In the same vein, the corresponding output feedback control problem has been studied in [71] for the systems with missing measurement and discrete distributed delays. Parallel to the control issues, the probabilitydependent gain-scheduling filtering problems have also been considered, and for systems with missing measurements, [70] has presented an elegant result, while a robust $H_{\infty}$ deconvolution filter has been designed in [73] to deal with the randomly occurring sensor delays.

\section{Latest Progress}

The probability-dependent gains-scheduling approach has been firstly proposed in [70], and it has been proven to be an effective one to deal with systems with randomly occurring incomplete information, it is now receiving increasing attention. For instance, in [112], under a guaranteed $H_{\infty}$ performance level, the probability-dependent gain-scheduling approach has been successfully applied to design a fault-tolerant controller for systems with randomly occurring actuator faults, while the probability-dependent gains-scheduling approach has been used in [72] in order to design a nonfragile gain-scheduled controller for systems with actuator uncertainties and random occurring sensor saturation. Furthermore, [51] has developed the probabilitydependent $H_{\infty}$ synchronization control scheme for a class of dynamical networks with randomly varying nonlinearities. 
The study on two-dimensional (2D) systems has recently attracted considerable attention due to their extensive applications in many engineering fields such as thermal process in chemical reactors, multidimensional digital filtering, and electron heating systems, [113-115]. In [116], the gain-scheduled control problem has been addressed for $2 \mathrm{D}$ discrete-time linear parameter-varying systems described by the Roesser model with matrices depending affinely on timevarying parameters which are measured on-line but their trajectory waveforms are not known in advance, and it is considered that the admissible values and variations of the parameters are confined to given intervals. Besides, the related guaranteed cost gain-scheduled control problem of 2D discrete-time linear parameter-varying systems has been investigated in [117].

The polynomial nonlinear systems are a rather general class of nonlinear systems. It is mainly about the nonlinearity disturbance in the nonlinear systems which can be approximated by polynomials via the Taylor expansion centered on the point we are interested in, and the introduced conservatism that came from the approximation error can be reduced by increasing the degree of the polynomials. Recently, the control and filtering issues for polynomial nonlinear systems have attracted some initial research attention. For instance, a nonlinear gain-scheduling output-feedback control problem has been addressed in [118] for polynomial nonlinear systems subject to actuator saturation.

Recently, in the gain-scheduled paradigm, the scheduling parameters with uncertainties have received scattered attention. For instance, in [119], the scheduling parameters are supposed to be provided with bounded proportional uncertainties; then, the corresponding $H_{\infty}$-type control problem is tackled and a sufficient condition for the resulting problem is given in terms of parameter-dependent LMIs. Moreover, the relationship of the proposed method and an existing method for tackling proportional uncertainties is clarified.

A limitation of the original gain-scheduling approaches is that the closed-loop stability can only be assured when the underlying parameters vary sufficiently slowly. A remedy exists but requires the implementation of possible solution of asymptotic Riccati equations (AREs) for an infinite number of different parameter values and the on-line solution of a Riccati differential equation (RDE) with time-varying coefficient matrices. The method in [120] avoids solving the RDE online; instead, it uses an explicit transient formula that looks up the predetermined solutions of the associated AREs at a finite set of given system operating points; furthermore, only a finite number of AREs are need to be solved to determine a finite set of controller gains.

There is a long history of gain-scheduling in applications areas. Recently, in [121], a robust control synthesis framework has been presented for switched direct current to DC-DC converters. The framework was based on an LMI formulation which can be solved automatically by efficient convex optimization algorithms. In [122], a control strategy based on gain-scheduling is proposed, which is able to regulate both stiffness and position at output link, and the gain-scheduled controller is designed based on a set of linear quadratic regulators, which are with inherent robustness properties and can accommodate significant variations in the actuation plant parameters.

\section{Conclusions and Future Work}

In this paper, we have summarized some recent advances on the gain-scheduled control and filtering for several kinds of systems with randomly occurring incomplete information. Several kinds of techniques related to gain-scheduled control and filtering algorithms have been surveyed. Next, the research and development of various system models have been reviewed, such as stochastic nonlinear systems, networked control systems, and linear parametervarying systems. Furthermore, the probability-dependent gain-scheduled control and filtering problems for systems with randomly occurring incomplete information have been introduced. To conclude this survey paper, based on the literature review, some related topics for the future research work are listed as follows.

(1) In engineering applications, there still exist many more complex yet important randomly occurring issues which, however, have not been considered. Therefore, it would be a promising research topic to discuss these new phenomena of incomplete information and establish a unified measurement model accounting for these issues simultaneously.

(2) In the existing literatures, a lot of results are based on the LMI conditions. While the interior-point LMI solvers are significantly faster than the classical convex optimization algorithms, it should be kept in mind that the complexity of LMI computations remains higher than that of solving, for example, a Riccati equation. For instance, problems with thousand design variables typically take over an hour on today's workstations. Therefore, another future research direction is to reduce the computation cost while keeping the desired performances.

(3) In case that multiple randomly occurring incomplete information appear simultaneously and influence each other in the same systems, the probabilitydependent gain-scheduling analysis and synthesis problem is a challenge, which constitutes one of the future research topics.

(4) When there is randomly occurring incomplete information in two-dimensional system, the probabilitydependent gain-scheduled control and filtering problems are potential research topics.

(5) An additional trend for future research is to discuss the probability-dependent gain-scheduled synchronization, control, and filtering problems for nonlinear stochastic complex networks with randomly occurring incomplete information.

\section{Conflict of Interests}

The authors declare that there is no conflict of interests regarding the publication of this paper. 


\section{Acknowledgments}

This work was supported in part by the National Natural Science Foundation of China under Grants 61074016, 61374039, 61304010, and 61329301; the Natural Science Foundation of Jiangsu Province of China under Grant BK20130766; the Program for Professor of Special Appointment (Eastern Scholar) at Shanghai Institutions of Higher Learning; the Program for New Century Excellent Talents in University under Grant NCET-11-1051, the Leverhulme Trust of the U.K., the Alexander von Humboldt Foundation of Germany.

\section{References}

[1] H. Gao and T. Chen, " $H_{\infty}$ estimation for uncertain systems with limited communication capacity," IEEE Transactions on Automatic Control, vol. 52, no. 11, pp. 2070-2084, 2007.

[2] Z. Shu, J. Lam, and J. Xiong, "Non-fragile exponential stability assignment of discrete-time linear systems with missing data in actuators," IEEE Transactions on Automatic Control, vol. 54, no. 3, pp. 625-630, 2009.

[3] Z. Wang, D. W. C. Ho, and X. Liu, "Variance-constrained filtering for uncertain stochastic systems with missing measurements," IEEE Transactions on Automatic Control, vol. 48, no. 7, pp. 1254-1258, 2003.

[4] B. Shen, Z. Wang, J. Liang, and Y. Liu, "Recent advances on filtering and control for nonlinear stochastic complex systems with incomplete information: a survey," Mathematical Problems in Engineering, vol. 2012, Article ID 530759, 16 pages, 2012.

[5] H. Dong, Z. Wang, X. Chen, and H. Gao, "A review on analysis and synthesis of nonlinear stochastic systems with randomly occurring incomplete information," Mathematical Problems in Engineering, vol. 2012, Article ID 416358, 15 pages, 2012.

[6] K. H. Ang, G. Chong, and Y. Li, "PID control system analysis, design, and technology," IEEE Transactions on Control Systems Technology, vol. 13, no. 4, pp. 559-576, 2005.

[7] S. R. Desai and R. Prasad, "A new approach to order reduction using stability equation and big bang big crunch optimization," Systems Science \& Control Engineering, vol. 1, no. 1, pp. 20-27, 2013.

[8] I. C. Trelea, "The particle swarm optimization algorithm: convergence analysis and parameter selection," Information Processing Letters, vol. 85, no. 6, pp. 317-325, 2003.

[9] D. E. Goldberg and J. H. Holland, "Genetic algorithms and machine learning," Machine Learning, vol. 3, no. 2, pp. 95-99, 1988.

[10] A. Mehrsai, H. R. Karimi, and K. D. Thoben, "Integration of supply networks for customization with modularity in cloud and make-to-upgrade strategy," Systems Science \& Control Engineering, vol. 1, no. 1, pp. 28-42, 2013.

[11] M. Armbrust, A. Fox, R. Griffith et al., "A view of cloud computing," Communications of the ACM, vol. 53, no. 4, pp. 5058, 2010.

[12] Q. Zhang, L. Cheng, and R. Boutaba, "Cloud computing: stateof-the-art and research challenges," Journal of Internet Services and Applications, vol. 1, no. 1, pp. 7-18, 2010.

[13] R. N. Langlois, "Modularity in technology and organization," Journal of Economic Behavior \& Organization, vol. 49, no. 1, pp. 19-37, 2002.
[14] J. Gao and H. M. Budman, "Design of robust gain-scheduled PI controllers for nonlinear processes," Journal of Process Control, vol. 15, no. 7, pp. 807-817, 2005.

[15] S. M. Shahruz, "Design of gain-scheduled PI-type compensators for linear parameter-varying systems," in Proceedings of the American Control Conference, vol. 5, pp. 3341-3342, Albuquerque, NM, USA, June 1997.

[16] V. Vesely and A. Ilka, "Gain-scheduled PID controller design," Journal of Process Control, vol. 23, no. 8, pp. 1141-1148, 2013.

[17] M. H. Nguyen, K. Tan, and C. Teo, "PID gain-scheduling by parametric model predictive control," in Proceedings of the IEEE/ASME International Conference on Advanced Intelligent Mechatronics, pp. 944-948, Wollongong, Austrilia, July 2013.

[18] T. Başar and P. Bernhard, $H_{\infty}$-Optimal Control and Related Minimax Design Problems: A Dynamic Game Approach, Springer, New York, NY, USA, 2008.

[19] G. Zames, "Feedback and optimal sensitivity: model reference transformations, multiplicative seminorms, and approximate inverses," IEEE Transactions on Automatic Control, vol. 26, no. 2, pp. 301-320, 1981.

[20] J. C. Doyle, K. Glover, P. P. Khargonekar, and B. A. Francis, "State-space solutions to standard $\mathrm{H}_{2}$ and $H_{\infty}$ control problems," IEEE Transactions on Automatic Control, vol. 34, no. 8, pp. 831-847, 1989.

[21] D. S. Bernstein and W. M. Haddad, "LQG control with an $H_{\infty}$ performance bound: a Riccati equation approach," IEEE Transactions on Automatic Control, vol. 34, no. 3, pp. 293-305, 1989.

[22] B. Halder, B. Hassibi, and T. Kailath, "Linearly combined suboptimal mixed $\mathrm{H}_{2} / \mathrm{H}_{\infty}$ controllers," in Proceedings of the 36th IEEE Conference on Decision and Control, pp. 434-439, San Diego, Calif, USA, December 1997.

[23] P. P. Khargonekar and M. A. Rotea, "Mixed $H_{2} / H_{\infty}$ control: a convex optimization approach," IEEE Transactions on Automatic Control, vol. 36, no. 7, pp. 824-837, 1991.

[24] W. Xie, " $H_{2}$ gain scheduled state feedback for LPV system with new LMI formulation," IEE Proceedings: Control Theory and Applications, vol. 152, no. 6, pp. 693-697, 2005.

[25] V.F. Montagner, R. C. L. F. Oliveira, and P. L. D. Peres, "Design of $H_{\infty}$ gain-scheduled controllers for linear time-varying systems by means of polynomial Lyapunov functions," in Proceedings of the 45th IEEE Conference on Decision and Control (CDC '06), pp. 5839-5844, San Diego, Calif, USA, December 2006.

[26] N. T. Hoang, H. D. Tuan, P. Apkarian, and S. Hosoe, "Gainscheduled filtering for time-varying discrete systems," IEEE Transactions on Signal Processing, vol. 52, no. 9, pp. 2464-2476, 2004.

[27] A. Karimi and Z. Emedi, " $H_{\infty}$ gain-scheduled controller design for rejection of time-varying narrow-band disturbances applied to a benchmark problem," European Journal of Control, vol. 19, no. 4, pp. 279-288, 2013.

[28] J. de Caigny, J. F. Camino, R. C. L. F. Oliveira, P. L. D. Peres, and J. Swevers, "Gain-scheduled $\mathrm{H}_{2} / \mathrm{H}_{\infty}$ control of discretetime polytopic time-varying systems," IET Control Theory \& Applications, vol. 4, no. 3, pp. 362-380, 2010.

[29] T. Takagi and M. Sugeno, "Fuzzy identification of systems and its applications to modeling and control," IEEE Transactions on Systems, Man and Cybernetics, vol. 15, no. 1, pp. 116-132, 1985.

[30] M. R. Moghanni-Bavil-Olyaei and A. Ghanbari, "Adaptive fuzzy terminal sliding mode control of MEMS Z-axis gyroscope with extended Kalman filter observer," Systems Science \& Control Engineering, vol. 2, no. 1, pp. 183-191, 2014. 
[31] R. M. Tong, "A control engineering review of fuzzy systems," Automatica, vol. 13, no. 6, pp. 559-568, 1977.

[32] M. Hamdy, "State observer based dynamic fuzzy logic system for a class of SISO nonlinear systems," International Journal of Automation and Computing, vol. 10, no. 2, pp. 118-124, 2013.

[33] K. Tanaka and M. Sugeno, "Stability analysis and design of fuzzy control systems," Fuzzy Sets and Systems, vol. 45, no. 2, pp. 135156, 1992.

[34] P. Balasubramaniam and T. Senthilkumar, "Delay-dependent robust stabilization and $H_{\infty}$ control for uncertain stochastic T-S fuzzy systems with discrete interval and distributed timevarying delay," International Journal of Automation and Computing, vol. 10, no. 1, pp. 18-31, 2013.

[35] F. Ahmida and E. H. Tissir, "Exponential stability of uncertain T-S fuzzy switched systems with time delay," International Journal of Automation and Computing, vol. 10, no. 1, pp. 32-38, 2013.

[36] Z.-Y. Zhao, M. Tomizuka, and S. Isaka, "Fuzzy gain-scheduling of PID controllers," IEEE Transactions on Systems, Man and Cybernetics, vol. 23, no. 5, pp. 1392-1398, 1993.

[37] P. Korba, R. Babuška, H. B. Verbruggen, and P. M. Frank, "Fuzzy gain scheduling: controller and observer design based on Lyapunov method and convex optimization," IEEE Transactions on Fuzzy Systems, vol. 11, no. 3, pp. 285-298, 2003.

[38] G. Wei, G. Feng, and Z. Wang, "Robust $H_{\infty}$ control for discrete-time fuzzy systems with infinite-distributed delays," IEEE Transactions on Fuzzy Systems, vol. 17, no. 1, pp. 224-232, 2009.

[39] A. Abdullah and M. Zribi, "Sensor fault tolerant control for a class of linear parameter varying systems with practical examples," IEEE Transactions on Industrial Electronics, vol. 60, no. 11, pp. 5239-5251, 2013.

[40] F. Wu and K. M. Grigoriadis, "LPV systems with parametervarying time delays: analysis and control," Automatica, vol. 37, no. 2, pp. 221-229, 2001.

[41] D. J. Stilwell and W. J. Rugh, "Stability and $L_{2}$ gain properties of LPV systems," Automatica, vol. 38, no. 9, pp. 1601-1606, 2002.

[42] O. Toker, "On the complexity of the robust stability problem for linear parameter varying systems," Automatica, vol. 33, no. 11, pp. 2015-2017, 1997.

[43] P. Apkarian, P. Gahinet, and G. Becker, "Self-scheduled $H_{\infty}$ control of linear parameter-varying systems: a design example," Automatica, vol. 31, no. 9, pp. 1251-1261, 1995.

[44] D. F. Coutinho, C. E. de Souza, and K. A. Barbosa, "Robust $H_{\infty}$ filter design for a class of discrete-time parameter varying systems," Automatica, vol. 45, no. 12, pp. 2946-2954, 2009.

[45] Y.-Y. Cao, Z. Lin, and Y. Shamash, "Set invariance analysis and gain-scheduling control for LPV systems subject to actuator saturation," Systems \& Control Letters, vol. 46, no. 2, pp. 137-151, 2002.

[46] C. E. de Souza and A. Trofino, "Gain-scheduled $H_{2}$ controller synthesis for linear parameter varying systems via parameterdependent Lyapunov functions," International Journal of Robust and Nonlinear Control, vol. 16, no. 5, pp. 243-257, 2006.

[47] F. Wang and V. Balakrishnan, "Improved stability analysis and gain-scheduled controller synthesis for parameter-dependent systems," IEEE Transactions on Automatic Control, vol. 47, no. 5, pp. 720-734, 2002.

[48] S. Zhou, B. Zhang, and W. X. Zheng, "Gain-scheduled $H_{\infty}$ filtering of parameter-varying discrete-time systems via parameterdependent Lyapunov functions," International Journal of Control, Automation and Systems, vol. 7, no. 3, pp. 475-479, 2009.
[49] W. J. Rugh and J. S. Shamma, "Research on gain scheduling," Automatica, vol. 36, no. 10, pp. 1401-1425, 2000.

[50] P. Apkarian, P. C. Pellanda, and H. D. Tuan, "Mixed $H_{2} / H_{\infty}$ multi-channel linear parameter-varying control in discrete time," Systems \& Control Letters, vol. 41, no. 5, pp. 333-346, 2000.

[51] L. Wang, G. Wei, and W. Li, "Probability-dependent $H_{\infty}$ synchronization control for dynamical networks with randomly varying nonlinearities," Neurocomputing, vol. 133, pp. 369-376, 2014.

[52] E. K. Boukas and Z. K. Liu, Deterministic and Stochastic Time Delay Systems, Birkhäauser, Boston, Mass, USA, 2002.

[53] W.-H. Chen, Z.-H. Guan, and X. Lu, "Delay-dependent exponential stability of uncertain stochastic systems with multiple delays: an LMI approach," Systems \& Control Letters, vol. 54, no. 6, pp. 547-555, 2005.

[54] H. Gao, J. Lam, and C. Wang, "Robust energy-to-peak filter design for stochastic time-delay systems," Systems \& Control Letters, vol. 55, no. 2, pp. 101-111, 2006.

[55] G. Wei, Z. Wang, and H. Shu, "Robust filtering with stochastic nonlinearities and multiple missing measurements," Automatica, vol. 45, no. 3, pp. 836-841, 2009.

[56] H. Shu and G. Wei, " $H_{\infty}$ analysis of nonlinear stochastic timedelay systems," Chaos, Solitons \& Fractals, vol. 26, no. 2, pp. 637647, 2005.

[57] K. El-Tawil and A. A. Jaoude, "Stochastic and nonlinear-based prognostic model," Systems Science \& Control Engineering, vol. 1, no. 1, pp. 66-81, 2013.

[58] M. Kermani and A. Sakly, "Stability analysis for a class of switched nonlinear time-delay systems," Systems Science \& Control Engineering, vol. 2, no. 1, pp. 80-89, 2014.

[59] Y. Chen and K. A. Hoo, "Stability analysis for closed-loop management of a reservoir based on identification of reducedorder nonlinear model," Systems Science \& Control Engineering, vol. 1, no. 1, pp. 12-19, 2013.

[60] Z. Wang, B. Shen, and X. Liu, " $H_{\infty}$ filtering with randomly occurring sensor saturations and missing measurements," Automatica, vol. 48, no. 3, pp. 556-562, 2012.

[61] L. Wang, G. Wei, and H. Shu, "State estimation for complex networks with randomly occurring coupling delays," Neurocomputing, vol. 122, pp. 513-520, 2013.

[62] Z. Wang, D. W. C. Ho, Y. Liu, and X. Liu, "Robust $H_{\infty}$ control for a class of nonlinear discrete time-delay stochastic systems with missing measurements," Automatica, vol. 45, no. 3, pp. 684-691, 2009.

[63] X. Wang, E. E. Yaz, and J. Long, "Robust and resilient state dependent control of continuous-time nonlinear systems with general performance criteria," Systems Science \& Control Engineering, vol. 2, no. 1, pp. 34-40, 2014.

[64] J. Hu, Z. Wang, H. Gao, and L. K. Stergioulas, "Extended Kalman filtering with stochastic nonlinearities and multiple missing measurements," Automatica, vol. 48, no. 9, pp. 20072015, 2012.

[65] D. Ding, Z. Wang, H. Dong, and H. Shu, "Distributed $H_{\infty}$ state estimation with stochastic parameters and nonlinearities through sensor networks: the finite-horizon case," Automatica, vol. 48, no. 8, pp. 1575-1585, 2012.

[66] Y. Yin, P. Shi, and F. Liu, "Gain-scheduled PI tracking control on stochastic nonlinear systems with partially known transition probabilities," Journal of the Franklin Institute, vol. 348, no. 4, pp. 685-702, 2011. 
[67] Y. Yin, P. Shi, and F. Liu, "Gain-scheduled robust fault detection on time-delay stochastic nonlinear systems," IEEE Transactions on Industrial Electronics, vol. 58, no. 10, pp. 4908-4916, 2011.

[68] P. Shi, Y. Yin, and F. Liu, "Gain-scheduled worst-case control on nonlinear stochastic systems subject to actuator saturation and unknown information," Journal of Optimization Theory and Applications, vol. 156, no. 3, pp. 844-858, 2013.

[69] Y. Liu, Y. Yin, F. Liu, P. Shi, and K. L. Teo, "Gain-scheduled $H_{\infty}$ control for nonlinear stochastic systems with mixed uncertainties," in Proceeding of the 8th IEEE Conference on Industrial Electronics and Applications, pp. 1544-1549, Melbourne, Australia, June 2013.

[70] G. Wei, Z. Wang, B. Shen, and M. Li, "Probability-dependent gain-scheduled filtering for stochastic systems with missing measurements," IEEE Transactions on Circuits and Systems II: Express Briefs, vol. 58, no. 11, pp. 753-757, 2011.

[71] W. Li, G. Wei, and L. Wang, "Probability-dependent static output feedback control for discrete-time nonlinear stochastic systems with missing measurements," Mathematical Problems in Engineering, vol. 2012, Article ID 696742, 15 pages, 2012.

[72] W. Li, G. Wei, H. R. Karimi, and X. Liu, "Non-fragile gainscheduled control for discrete-time stochastic systems with randomly occurring sensor saturations," Abstract and Applied Analysis, vol. 2013, Article ID 629621, 10 pages, 2013.

[73] Y. Luo, G. Wei, H. R. Karimi, and L. Wang, "Deconvolution filtering for nonlinear stochastic systems with randomly occurring sensor delays via probability-dependent method," Abstract and Applied Analysis, vol. 2013, Article ID 814187, 12 pages, 2013.

[74] G. Wei, Z. Wang, and B. Shen, "Probability-dependent gainscheduled control for discrete stochastic delayed systems with randomly occurring nonlinearities," International Journal of Robust and Nonlinear Control, vol. 23, no. 7, pp. 815-826, 2013.

[75] R. M. Murray, K. J. Astrom, and S. P. Boyd, "Future directions in control in an information-rich world," IEEE Control Systems, vol. 23, no. 2, pp. 20-33, 2003.

[76] J. P. Hespanha, P. Naghshtabrizi, and Y. Xu, "A survey of recent results in networked control systems," Proceedings of the IEEE, vol. 95, no. 1, pp. 138-162, 2007.

[77] T. C. Yang, "Networked control system: a brief survey," IEE Proceedings: Control Theory and Applications, vol. 153, no. 4, pp. 403-412, 2006.

[78] S. Zampieri, "Trends in networked control systems," in Proceedings of the 17th World Congress The International Federation of Automatic Control, pp. 2886-2894, Seoul, Republic of Korea, July 2008.

[79] M. Liu, D. W. C. Ho, and Y. Niu, "Stabilization of Markovian jump linear system over networks with random communication delay," Automatica, vol. 45, no. 2, pp. 416-421, 2009.

[80] L. Zhang, Y. Shi, T. Chen, and B. Huang, "A new method for stabilization of networked control systems with random delays," IEEE Transactions on Automatic Control, vol. 50, no. 8, pp. 11771181, 2005.

[81] Y.-C. Tian and D. Levy, "Compensation for control packet dropout in networked control systems," Information Sciences, vol. 178 , no. 5, pp. 1263-1278, 2008.

[82] W. Zhang, M. S. Branicky, and S. M. Phillips, "Stability of networked control systems," IEEE Control Systems Magazine, vol. 21, no. 1, pp. 84-99, 2001.

[83] W.-A. Zhang and L. Yu, "Output feedback stabilization of networked control systems with packet dropouts," IEEE Transactions on Automatic Control, vol. 52, no. 9, pp. 1705-1710, 2007.
[84] X.-M. Tang and B.-C. Ding, "Design of networked control systems with bounded arbitrary time delays," International Journal of Automation and Computing, vol. 9, no. 2, pp. 182-190, 2012.

[85] S. Liu, X. P. Liu, and A. El Saddik, "Modeling and dynamic gain-scheduling for networked systems with bounded packet losses," in Proceedings of the IEEE International Workshop on Measurements and Networking, pp. 135-139, Anacapri, Italy, October 2011.

[86] H. Li, Z. Sun, M.-Y. Chow, and F. Sun, "Gain-scheduling-based state feedback integral control for networked control systems," IEEE Transactions on Industrial Electronics, vol. 58, no. 6, pp. 2465-2472, 2011.

[87] M. Tai and K. Uchida, "Gain scheduled output feedback control of discrete-time networked systems," in Proceedings of the IEEE International Conference on Control Applications (CCA '07), pp. 59-64, Singapore, October 2007.

[88] Y.-B. Zhao, J. Kim, and G.-P. Liu, "Offline model predictive control-based gain scheduling for networked control systems," IET Control Theory \& Applications, vol. 6, no. 16, pp. 2585-2591, 2012.

[89] Y. Ji and H. J. Chizeck, "Controllability, stabilizability, and continuous-time Markovian jump linear quadratic control," IEEE Transactions on Automatic Control, vol. 35, no. 7, pp. 777788, 1990.

[90] P.-F. Zhou, Y.-Y. Wang, Q.-B. Wang, J.-A. Chen, and D.-P. Duan, "Stability and passivity analysis for Lur'e singular systems with Markovian switching," International Journal of Automation and Computing, vol. 10, no. 1, pp. 79-84, 2013.

[91] L. Wu, P. Shi, and H. Gao, "State estimation and slidingmode control of Markovian jump singular systems," IEEE Transactions on Automatic Control, vol. 55, no. 5, pp. 1213-1219, 2010.

[92] H. Gao, C. Wang, and J. Wang, "On $H_{\infty}$ performance analysis for continuous-time stochastic systems with polytopic uncertainties," Circuits, Systems, and Signal Processing, vol. 24, no. 4, pp. 415-429, 2005.

[93] P. Shi, M. Mahmoud, S. K. Nguang, and A. Ismail, "Robust filtering for jumping systems with mode-dependent delays," Signal Processing, vol. 86, no. 1, pp. 140-152, 2006.

[94] P. Shi, M. S. Mahmoud, J. Yi, and A. Ismail, "Worst case control of uncertain jumping systems with multi-state and input delay information," Information Sciences, vol. 176, no. 2, pp. 186-200, 2006.

[95] L. Ma, F. Da, and K.-J. Zhang, "Exponential $H_{\infty}$ filter design for discrete time-delay stochastic systems with markovian jump parameters and missing measurements," IEEE Transactions on Circuits and Systems I: Regular Papers, vol. 58, no. 5, pp. 9941007, 2011.

[96] H. Liu, F. Sun, K. He, and Z. Sun, "Design of reduced-order $H_{\infty}$ filter for Markovian jumping systems with time delay," IEEE Transactions on Circuits and Systems II: Express Briefs, vol. 51, no. 11, pp. 607-612, 2004.

[97] D. Yue and Q.-L. Han, "Delay-dependent exponential stability of stochastic systems with time-varying delay, nonlinearity, and Markovian switching," IEEE Transactions on Automatic Control, vol. 50, no. 2, pp. 217-222, 2005.

[98] C. Yuan and X. Mao, "Robust stability and controllability of stochastic differential delay equations with Markovian switching," Automatica, vol. 40, no. 3, pp. 343-354, 2004.

[99] L. Zhang and E.-K. Boukas, "Mode-dependent $H_{\infty}$ filtering for discrete-time Markovian jump linear systems with partly 
unknown transition probabilities," Automatica, vol. 45, no. 6, pp. 1462-1467, 2009.

[100] L. Zhang and J. Lam, "Necessary and sufficient conditions for analysis and synthesis of Markov jump linear systems with incomplete transition descriptions," IEEE Transactions on Automatic Control, vol. 55, no. 7, pp. 1695-1701, 2010.

[101] Y. Yin, F. Liu, and Y. Shi, "Gain scheduled $L_{2}-L_{\infty}$ filtering for neutral systems with jumping and time-varying parameters," Journal of Control Theory and Applications, vol. 10, no. 1, pp.118$123,2012$.

[102] H. Ahmad and T. Namerikawa, "Extended Kalman filter-based mobile robot localization with intermittent measurements," Systems Science \& Control Engineering, vol. 1, no. 1, pp. 113-126, 2013.

[103] Z. Wang, H. Dong, B. Shen, and H. Gao, "Finite-horizon $H_{\infty}$ filtering with missing measurements and quantization effects," IEEE Transactions on Automatic Control, vol. 58, no. 7, pp. 17071718, 2013.

[104] Z. Wang, B. Shen, H. Shu, and G. Wei, "Quantized $H_{\infty}$ control for nonlinear stochastic time-delay systems with missing measurements," IEEE Transactions on Automatic Control, vol. 57, no. 6, pp. 1431-1444, 2012.

[105] J. Liang, F. Sun, and X. Liu, "Finite-horizon $H_{\infty}$ filtering for time-varying delay systems with randomly varying nonlinearities and sensor saturations," Systems Science \& Control Engineering, vol. 2, no. 1, pp. 108-118, 2014.

[106] E. Tian, P. Chen, and G. Zhou, "Fault tolerant control for discrete networked control systems with random faults," International Journal of Control, Automation and Systems, vol. 10, no. 2, pp. 444-448, 2012.

[107] G. Wei, L. Wang, and W. Li, "Fault-tolerant control for discretetime stochastic systems with randomly occurring faults," in Proceedings of the 31st Chinese Control Conference, pp. 15351540, Hefei, China, July 2012.

[108] R. Yang, P. Shi, and G.-P. Liu, "Filtering for discrete-time networked nonlinear systems with mixed random delays and packet dropouts," IEEE Transactions on Automatic Control, vol. 56, no. 11, pp. 2655-2660, 2011.

[109] B. Shen, Z. Wang, H. Shu, and G. Wei, "Robust $H_{\infty}$ finitehorizon filtering with randomly occurred nonlinearities and quantization effects," Automatica, vol. 46, no. 11, pp. 1743-1751, 2010.

[110] H. Dong, Z. Wang, and H. Gao, "Distributed $H_{\infty}$ filtering for a class of Markovian jump nonlinear time-delay systems over lossy sensor networks," IEEE Transactions on Industrial Electronics, vol. 60, no. 10, pp. 4665-4672, 2013.

[111] J. Hu, Z. Wang, B. Shen, and H. Gao, "Quantised recursive filtering for a class of nonlinear systems with multiplicative noises and missing measurements," International Journal of Control, vol. 86, no. 4, pp. 650-663, 2013.

[112] G. Wei, L. Wang, and F. Han, "A gain-scheduled approach to fault-tolerant control for discrete-time stochastic delayed systems with randomly occurring actuator faults," Systems Science \& Control Engineering, vol. 1, no. 1, pp. 82-90, 2013.

[113] T. Kaczorek, Two-Dimensional Linear Systems, Springer, Berlin, Germany, 1985.

[114] D. N. Godard, "Self-recovering equalization and carrier tracking in two-dimensional data communication systems," IEEE Transactions on Communications, vol. 28, no. 11, pp. 1867-1875, 1980.
[115] R. P. Roesser, "A discrete state-space model for linear image processing," IEEE Transactions on Automatic Control, vol. 20, no. 1, pp. 1-10, 1975.

[116] C. E. de Souza and J. Osowsky, "Gain-scheduled control of twodimensional discrete-time linear parameter-varying systems in the Roesser model," Automatica, vol. 49, no. 1, pp. 101-110, 2013.

[117] C. E. de Souza and J. Osowsky, "Guaranteed cost gain-scheduled control of two-dimensional discrete-time linear parametervarying systems," in Proceedings of the 7th International Workshop on Multidimensional Systems, pp. 5-7, Oitiers, France, September 2011.

[118] F. Wu and S. Hays, "Nonlinear gain-scheduling output-feedback control for polynomial nonlinear systems subject to actuator saturation," International Journal of Control, vol. 86, no. 9, pp. 1607-1619, 2013.

[119] M. Sato and D. Peaucelle, "Gain-scheduled output-feedback controllers using inexact scheduling parameters for continuous-time LPV systems," Automatica, vol. 49, no. 4, pp. 1019-1025, 2013.

[120] A. Dehghani, M. C. Rotkowitz, B. D. O. Anderson, and S. H. Cha, "Simplified rapid switching gain scheduling for a class of LPV systems," IEEE Transactions on Automatic Control, vol. 57, no. 10, pp. 2633-2639, 2012.

[121] C. Olalla, R. Leyva, I. Queinnec, and D. Maksimovic, "Robust gain-scheduled control of switched-mode DC-DC converters," IEEE Transactions on Power Electronics, vol. 27, no. 6, pp. 30063019, 2012.

[122] I. Sardellitti, G. A. Medrano-Cerda, N. Tsagarakis, A. Jafari, and D. G. Caldwell, "Gain-scheduling control for a class of variable stiffness actuators based on lever mechanisms," IEEE Transactions on Robotics, vol. 29, no. 3, pp. 797-798, 2013. 


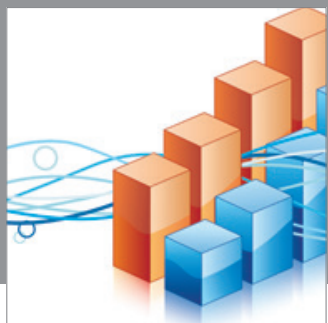

Advances in

Operations Research

mansans

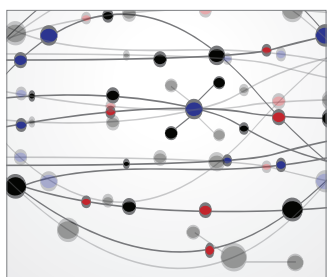

The Scientific World Journal
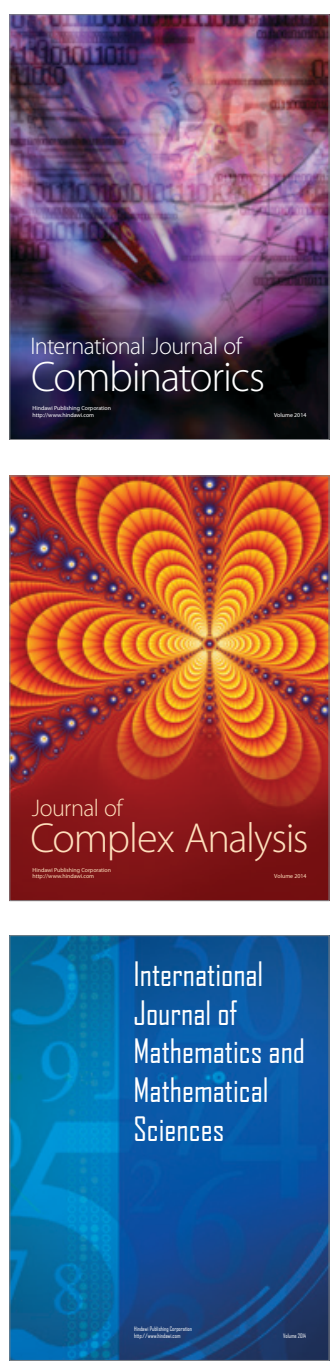
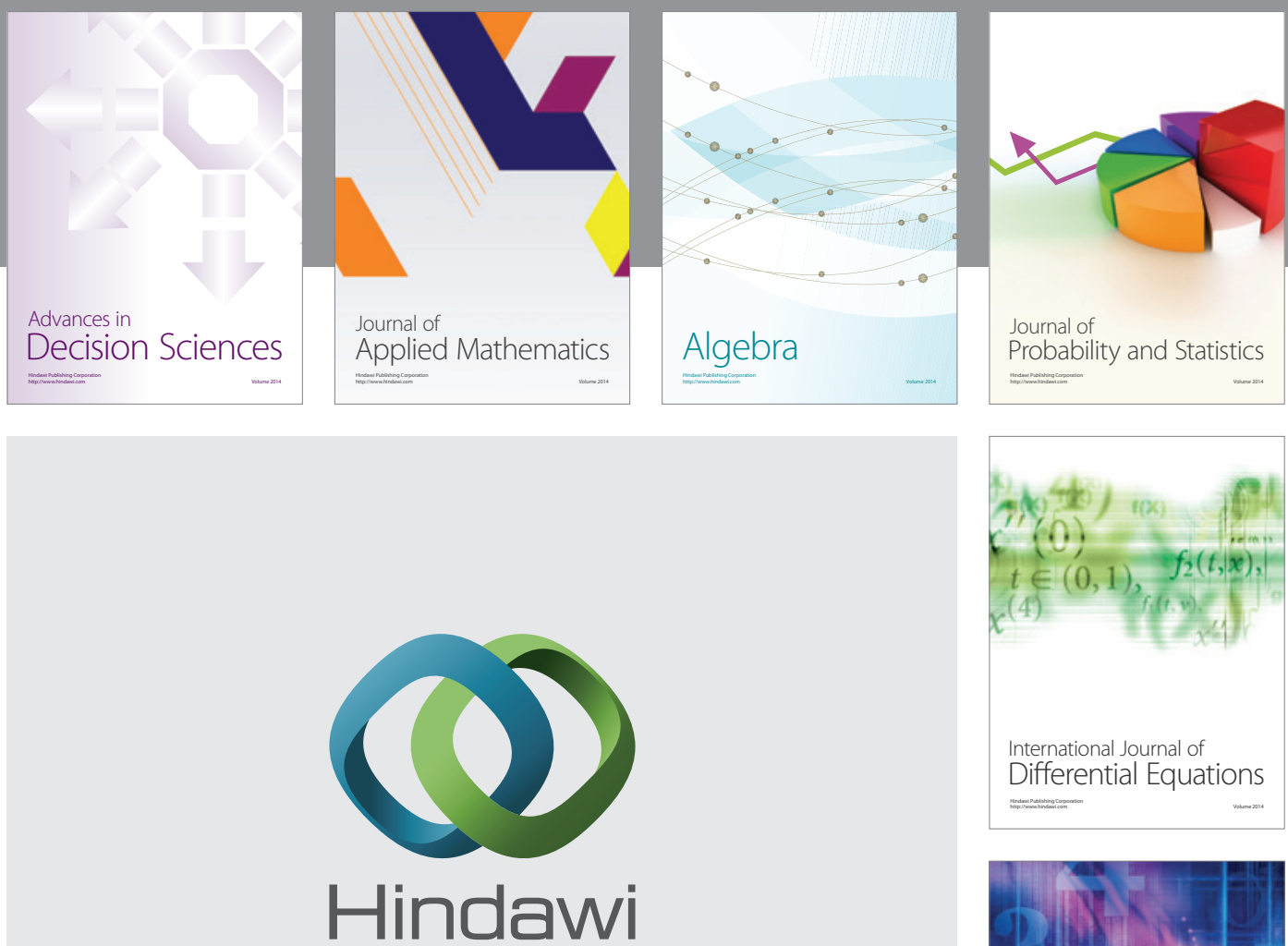

Submit your manuscripts at http://www.hindawi.com
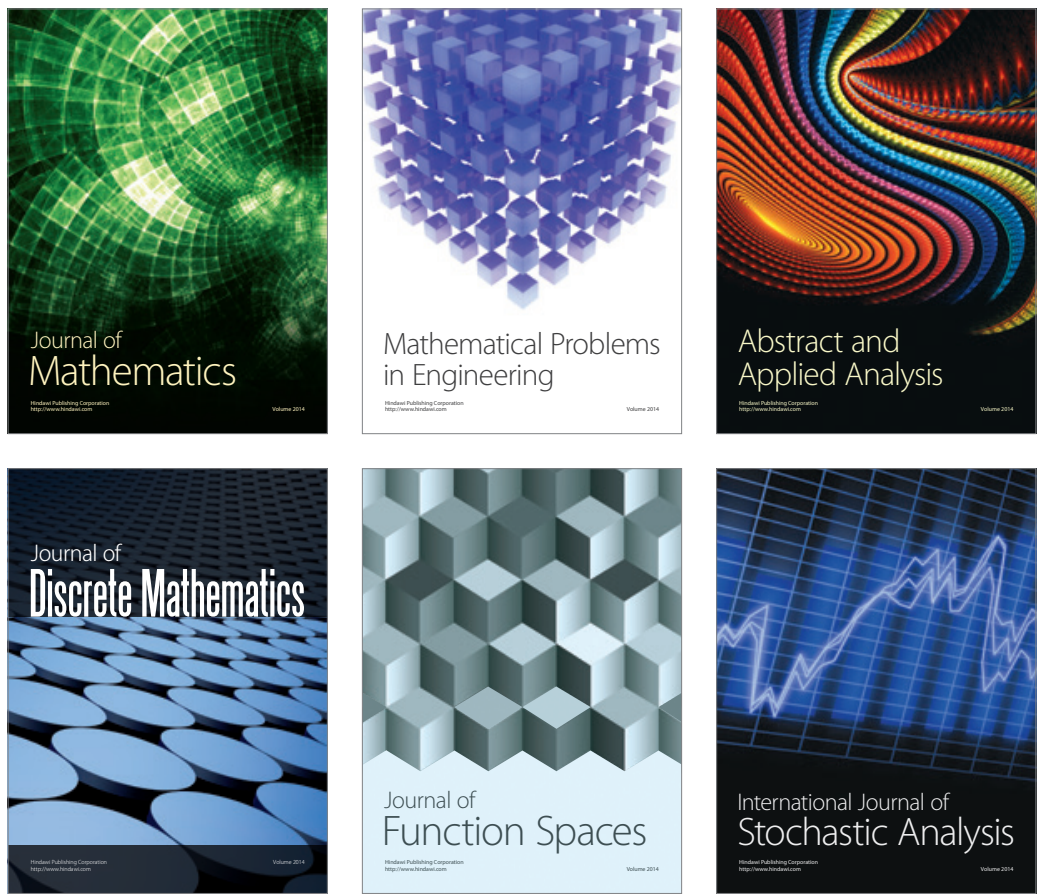

Journal of

Function Spaces

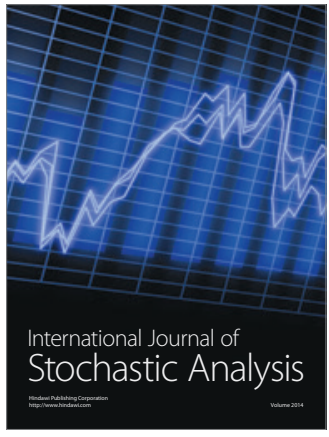

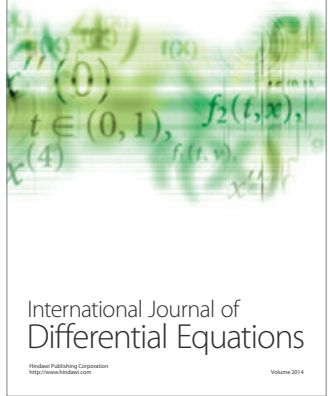
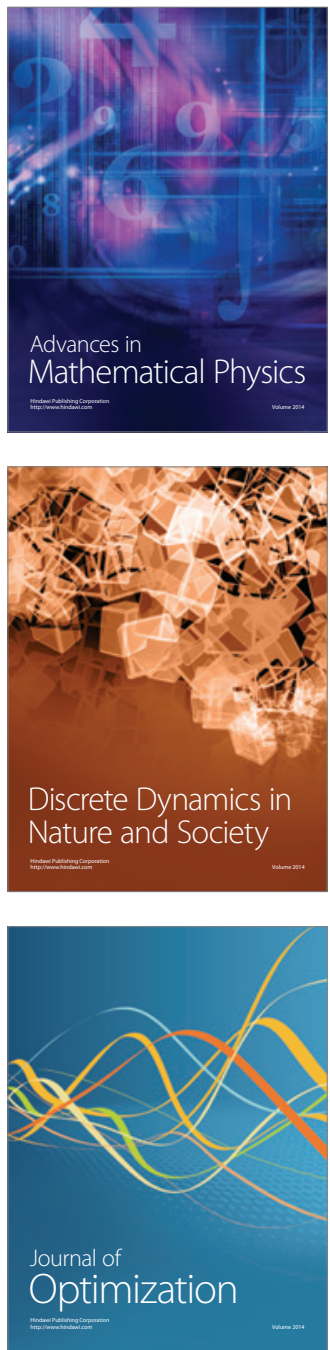